\title{
Topografia do cone medular do cachorro-do-mato-de-orelhas-pequenas (Atelocynus microtis Sclater, 1882): relato de caso
}

\author{
Kylma Lorena Saldanha \\ Érika Branco* \\ Ana Rita de Lima \\ Universidade Federal Rural da Amazônia \\ Avenida Presidente Tancredo Neves, 2501, CEP 66077-530, Belém - PA, Brasil \\ *Autor para correspondência \\ ebranco.ufra@gmail.com
}

Submetido em 24/05/2011

Aceito para publicação em 16/08/2011

\section{Resumo}

A anestesia epidural é uma das técnicas anestésicas mais utilizadas devido à boa margem de segurança, eficiência e rapidez na aplicação. Decorrente de sua importância faz-se necessário ter conhecimento preciso da topografia do cone medular das espécies. Portanto, este trabalho teve como objetivo descrever a topografia do cone medular do cachorro-do-mato-de-orelhas-pequenas (Atelocynus microtis), com o propósito de fornecer dados anatômicos úteis para a prática de anestesias epidurais. Foi utilizado um exemplar jovem, macho, após morte natural, proveniente da Área de Mina Bauxita de Paragominas-PA, o qual foi fixado e armazenado em solução aquosa de formaldeído a 10\%. O animal foi dissecado mediante afastamento da pele e da musculatura epiaxial, procedendo-se a secção e remoção dos arcos vertebrais favorecendo a visualização da medula espinhal. O Atelocynus microtis apresentou sete vértebras lombares e três sacrais. Após a individualização das estruturas anatômicas de interesse, foram tomadas as mensurações do cone medular com o auxílio de paquímetro. A intumescência lombar localizou-se entre as vértebras L3 e L6, o cone medular foi observado em nível da sétima vértebra lombar (L7) e seu ápice na terceira vértebra sacral (S3) totalizando um comprimento de 3,9cm acompanhado pela cauda equina. Assim, sugerimos a região sacrocaudal como o local mais apropriado para realização de anestesia epidural no Atelocynus microtis.

Palavras-chave: Atelocynus microtis, Cone medular; Topografia vértebro-medular

\section{Abstract}

Topography of the medullar cone in the short-eared dog (Atelocynus microtis Sclater, 1882): Case report. Epidural anesthesia is one of the most common anesthetic techniques used because it is safe, efficient and can be quickly implemented. However, for each species undergoing this procedure, precise knowledge of the topography of the medullar cone is necessary. Therefore, this study aimed to describe the topography of the medullar cone of the short-eared dog (Atelocynus microtis), in order to provide relevant anatomical data for the practice of epidural anesthesia on this species. We used an exemplary young male, from the Bauxite Mine area (Paragominas, PA), which died of natural causes and was fixed and stored in 10\% aqueous formaldehyde. The 
animal was dissected by removing the skin and epiaxial musculature, followed by the section and removal of vertebral arches, which exposed the medullar cone. The specimen studied had seven lumbar and three sacral vertebras. After the separation of the anatomical structures, the conus medullaris was measured with a digital caliper. The lumbar intumescence was located between L3 and L6, the medullar cone was observed in L7 and its apex in the $\mathrm{S} 3$, and had a total length of $3.9 \mathrm{~cm}$. We suggest that the sacrocaudal region is the most appropriate place to perform epidural anesthesia in Atelocynus microtis.

Key words: Atelocynus microtis, Medullar cone, Vertebro-medullar topography

\section{Introdução}

Conhecido popularmente como cachorro-domato-de-orelhas-pequenas ou de raposa-de-orelhascurtas, Atelocynus microtis é um mamífero carnívoro pertencente à família Canidae. Esta espécie é a única de seu gênero e a mais rara de canídeos sul-americanos (BERTA, 1986; KOESTER et al., 2008).

O cachorro-do-mato-de-orelhas-pequenas apresenta a cabeça grande, membros delgados relativamente curtos e cauda longa e peluda com uma lista avermelhada contínua na parte ventral. Na região dorsal do corpo, incluindo a cauda, nota-se uma estreita faixa negra, de forma que somente ao redor da região pubiana e na parte ventral da base da cauda é que encontramos áreas de pelagem clara com mechas brancas ou amareladas (BERTA, 1986). Sua altura pode chegar a $25 \mathrm{~cm}$, com comprimento variando entre 42 e $100 \mathrm{~cm}$, cauda medindo $30 \mathrm{~cm}$ e peso corpóreo de aproximadamente $10 \mathrm{~kg}$ (MOSKOSKI, 2010).

Geograficamente, o A. microtis está distribuído na América do Sul, estendendo-se desde a Colômbia, Bolívia, Equador, Peru e Brasil, até o norte do Mato Grosso (CUBAS et al., 2007), além de estar disperso, por grande parte da bacia Amazônica, chegando à bacia do rio Paraguai, através do rio Araguaia-Tocantins. Esta espécie possui dieta carnívora e apresenta hábitos noturnos e solitários, sendo considerado o menos gregário dos canídeos sul-americanos (KOESTER et al., 2008).

Poucos são os estudos realizados com A. microtis, principalmente sobre a morfologia do sistema nervoso dessa espécie. Este conhecimento é de suma importância para subsidiar procedimentos clínicos e cirúrgicos no âmbito da medicina veterinária de animais silvestres, tais como punção medular, localização de lesões nervosas centrais em níveis vertebrais específicos e bloqueios anestésicos (peridurais), sendo esta a técnica anestésica mais utilizada devido à sua segurança, eficiência e rapidez de aplicação (FANTONI; CORTOPASSI, 2002; DYCE et al., 2004).

Em busca de conhecimentos em relação às bases anatômicas do cone medular, este trabalho tem por objetivo oferecer dados relativos à topografia do cone medular dessa espécie indo ao encontro do interesse da medicina veterinária de animais selvagens, sobre a abordagem de procedimentos anestésicos, que poderão ser utilizados nestes animais.

\section{Material e Métodos}

Estudou-se um exemplar de cachorro-do-matode-orelhas-pequenas (Atelocynus microtis), jovem, macho, que foi a óbito por causas naturais, proveniente de Paragominas-PA, Brasil / Empresa Terra LTDA, sob autorização SEMA-PA No 455/2009 e 522/2009 e doado ao Laboratório de Pesquisa Morfológica Animal (LaPMA) da Universidade Federal Rural da Amazônia - UFRA.

$\mathrm{O}$ animal foi fixado com solução aquosa de formol a $10 \%$ por meio de perfusões intramusculares em todas as partes moles e cavidades corporais, e posteriormente armazenado em cuba plástica por um período de sete dias contendo o mesmo fixador.

Para a visualização da medula espinhal e de seus componentes, o animal foi submetido à dissecação realizando-se incisão e rebatimento da pele, da tela subcutânea e da musculatura na linha mediana dorsal das vértebras lombares e em toda a região das vértebras sacrais. Em seguida, os arcos vertebrais foram removidos através do uso de costótomo, revelando as meninges e a 
medula espinhal. Por fim, identificaram-se as vértebras torácicas e sacrais, o cone medular e a intumescência lombar seguido de mensuração com uso de paquímetro digital Dc-6 Western.

Toda nomenclatura adotada foi baseada na International Committee on Veterinary Gross Anatomical Nomenclature (2005).

\section{Resultados}

Após a dissecação da região lombo-sacral, a qual era formada por sete vértebras lombares e três vértebras sacrais, revelou-se a intumescência lombar, o cone medular e a cauda equina.

A intumescência lombar, ponto de delimitação da base do cone medular, apresentou-se entre a L3 e L6. Quanto à topografia do cone medular, observamos que este se localizou entre L7 (base) e S3 (ápice), revelando um comprimento de $3,9 \mathrm{~cm}$, sendo seguido pela cauda equina (Figura 1).

\section{Discussão}

Para diferentes espécies de animais domésticos ou selvagens, diversos autores têm se reportado à topografia do cone medular, tanto pela importância do tema quando relacionado a técnicas de práticas anestésicas epidurais, quanto pelo interesse da anatomia comparativa.

Comparando a esquelotopia do cone medular do cachorro-do-mato-de-orelhas-pequenas (base em
L7 e ápice em S3) com outros carnívoros selvagens como o lobo-guará (base do cone em L5 e ápice em L6) (MACHADO et al., 2002), gato mourisco (base do cone na L6 e ápice na S2) (CARVALHO et al., 2003), o lobo-marinho (base do cone na T5 e ápice na T6) (MACHADO et al., 2003), a ariranha (base do cone na L2 e ápice podendo se estender até L4) (MACHADO et al., 2009a), o quati (base do cone em torno da L5 e L6 e ápice no final da S3) (GREGORES et al., 2010), e a jaguatirica (base do cone ao nível da L4 e ápice ao final da S3) (BRIGIDA et al., 2010), podemos notar que estes outros carnívoros apresentam topografia do cone medular diferente, independentemente de pertencerem à mesma ordem.

Em carnívoros domésticos, como os cães, a base do cone medular apresenta-se, mais cranialmente, entre as vértebras L3 e L4 (SANTIAGO, 1974; KÖNIG; LIEBICH, 2004). Nos gatos domésticos (SILVA et al., 2009), a localização do cone medular é semelhante ao encontrado no Atelocynus microtis, situando-se entre as vértebras L5 (base) e S2 (ápice).

No grupo de alguns mamíferos não carnívoros, como caprino (base entre L6 ou entre L5 e L6 e seu ápice em torno da $\mathrm{S} 1$ e da S3) (SANTOS et al., 2001), paca (base em L5 e ápice em S1) (SCAVONE et al., 2007), ratão-do-banhado (base em torno da L3 e L5 e seu ápice ao nível da L5) (MACHADO et al., 2009b) e o xenartra, tamanduá-mirim (base do cone na L1 e ápice entre as vértebras L5 e L6) (MACHADO; SANTOS, 2008), observou-se que o cone medular destas espécies é relativamente maior do que a espécie alvo deste estudo.

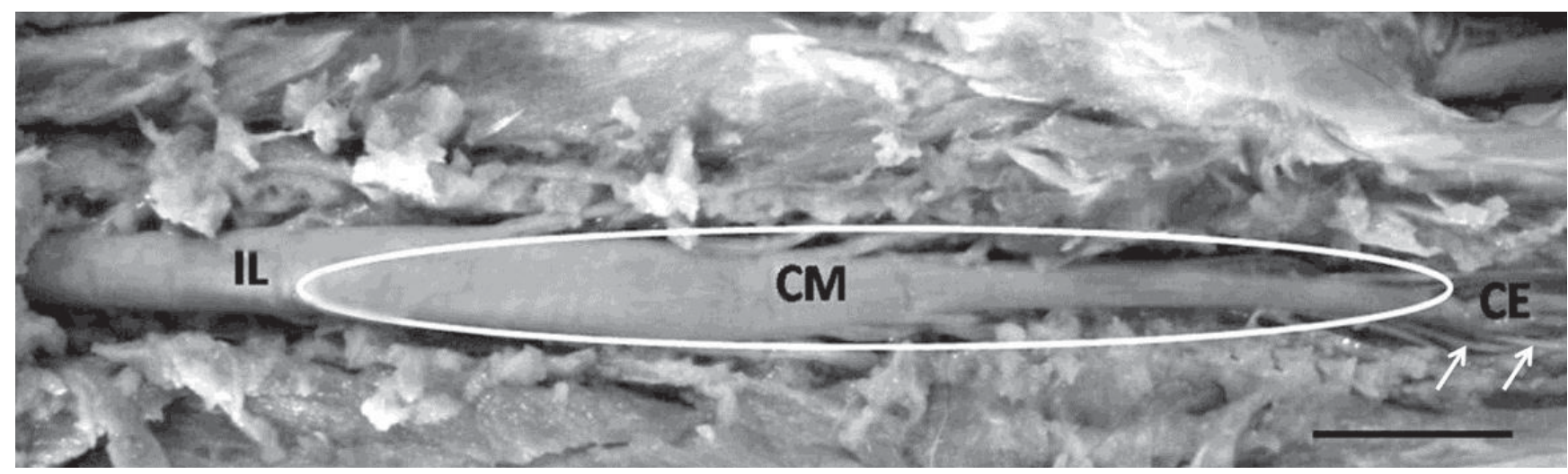

FIGURA 1: Fotografia da vista dorsal da porção terminal da medula espinal de Atelocynus microtis, destacando-se intumescência lombar (IL); cone medular (CM) (círculo) e cauda equina (CE) com filamentos terminais (setas). Escala de Barra: 0,5cm. 
Quanto ao comprimento total do cone medular, no cachorro-do-mato-de-orelhas-pequenas, notamos que esta espécie é a que tem menor extensão $(3,9 \mathrm{~cm})$ em relação aos demais carnívoros selvagens aqui citados, no qual podemos destacar o lobo-guará $(6,5 \mathrm{~cm})$ (MACHADO et al., 2002), gato mourisco $(5,0 \mathrm{~cm})$ (CARVALHO et al., 2003), lobo-marinho $(4,3 \mathrm{~cm})$ (MACHADO et al., 2003$)$, ariranha $(5,5 \mathrm{~cm})$ (MACHADO et al., 2009a), quati $(5,2$ a $5,8 \mathrm{~cm})$ (GREGORES et al., 2010), jaguatirica $(8,5 \mathrm{~cm})$ (BRÍGIDA et al., 2010) e o gato doméstico $(5,08 \mathrm{~cm})$ (SILVA et al., 2009).

Em relação a outros mamíferos não carnívoros, notamos que a mensuração do cone medular do cachorrodo-mato-de-orelhas-pequenas encontra-se na média de comprimento $(3,9 \mathrm{~cm})$, uma vez que o ovino (sem raça definida) apresenta, dentre todos os citados, o maior cone medular, tendo média de $8,22 \mathrm{~cm}$ (SANTOS; LIMA, 2000), seguido pelo tamanduá-mirim (6,5 a $7,0 \mathrm{~cm}$ ) (MACHADO; SANTOS, 2008), caprinos da raça Saanen $(3,44 \mathrm{~cm})$ (SANTOS et al., 2001$)$, paca $(3,39 \mathrm{~cm})$ (SCAVONE et al., 2007), e ratão-do-banhado $(2 \mathrm{~cm})$ (MACHADO et al., 2009b), sendo este com o menor comprimento de cone medular.

Concluímos que a base do cone medular do Atelocynus microtis fica localizada entre a sétima vértebra lombar (L7) e a terceira vértebra sacral (S3), medindo $3,9 \mathrm{~cm}$ de comprimento. Com base nos dados, sugere-se para a realização de anestesia epidural nesta espécie, a região sacrocaudal.

\section{Agradecimentos}

À bióloga Ana Carla Barros de Souza e à médica veterinária Luiza Corrêa Pereira, ambas da Empresa Terra, da Área de Mina Bauxita - Paragominas - PA, pela doação do material.

\section{Referências}

BERTA, A. Atelocynus microtis. Mammalian Species, Washington, n. 256, p. 1-3, 1986.

BRIGIDA, S. S. S.; BRANCO, E.; PINHEIRO, L. L.; MARTINS, D. M.; ARAÚJO, E. B.; MELUL, R.; JR., A. C. C. L.; MENESES,
A. M. C.; SOUZA, A. C. B.; PEREIRA, L. C.; FIORETTO, E. T.; LIMA, A. R. Topografia do cone medular da jaguatirica (Leopardus pardalis). Acta Veterinaria Brasilica, Mossoró, v. 4, n. 1, p. 5154, 2010.

CARVALHO, S. F. M.; SANTOS, A. L. Q.; AVILA JUNIOR, R. H.; ANDRADE, M. B.; MAGALHÃES, L. M.; MORAES, F. M.; RIBEIRO, P. I. R. Topografia do cone medular em um gato mourisco, Herpailurus yagouaroundi (Severtzo, 1858) (Felidae). Archives of Veterinary Science, Curitiba, v. 8, n. 2, p. 35-38, 2003.

CUBAS, Z. S.; SILVA, J. C. R.; CATÃO-DIAS, J. L. Tratado de animais selvagens - Medicina Veterinária. São Paulo: Roca, 2007. $492 \mathrm{p}$.

DYCE, K. M.; SACK, W. O.; WENSING, C. J. G. Tratado de anatomia veterinária. 3. ed. Rio de Janeiro: Guanabara Koogan, $2004.813 \mathrm{p}$.

FANTONI, D. T.; CORTOPASSI, S. R. G. Anestesia em cães e gatos. 2. ed. São Paulo: Roca, 2002. 389 p.

GREGORES, G. B.; BRANCO, E.; CARVALHO, A. F.; SARMENTO, C. A. P.; OLIVEIRA, P. C.; FERREIRA, G. J.; CABRAL, R.; FIORETO, E. T.; MIGLINO, M. A.; CORTOPASSI, S. R. G. Topografia do cone medular do quati (Nasua nasua, Linnaeus 1766). Biotemas, Florianópolis, v. 23, n. 2, p. 173-176, 2010.

INTERNATIONAL COMMITTEE ON VETERINARY GROSS ANATOMICAL NOMENCLATURE. Nomina anatômica veterinária. 5. ed. Knoxville: World Association on Veterinary Anatomist, 2005. $190 \mathrm{p}$.

KOESTER, A. D.; AZEVEDO, C. R.; VOGLIOTTI, A.; DUARTE, J. M. B. Ocorrência de Atelocynus microtis (Sclater, 1882) na Floresta Nacional do Jamari, estado de Rondônia. Biota Neotropica, Campinas, v. 8, n. 4, p. 231-234, 2008.

KÖNIG, H. E.; LIEBICH, H. G. Tecido nervoso. In: KÖNIG, H. E.; LIEBICH, H. G. (Ed.). Anatomia dos animais domésticos: Texto e atlas colorido. Porto Alegre: Artmed, 2004. p. 205-206.

MACHADO, G. V.; CAL, J. A.; BIRCK, A. J. Topografia do cone medular no ratão-do-banhado (Myocastor coypus Molina, 1782 Rodentia: Mammalia). Biotemas, Florianópolis, v. 22, n. 2, p. 117120, 2009b.

MACHADO, G. V.; LESNAU, G. G.; BIRCK, A. J. Topografia do cone medular no lobo marinho (Arctocephalus australis Zimmermann, 1803). Arquivos de Ciências Veterinárias e Zoologia da UNIPAR, Umuarama, v. 6, n. 1, p. 11-14, 2003.

MACHADO, G. V.; ROSAS, F. C. W.; LAZZARINI, S. M. Topografia do cone medular na ariranha (Pteronura brasiliensis Zimmermann, 1780). Ciência Animal Brasileira, Goiânia, v. 10, n. 1, p. 301-305, 2009a.

MACHADO, V. M.; FONSECA, C. C.; NEVES, M. T. D.; PAULA, T. A. R.; BENJAMIN, L. A. Topografia do cone medular no loboguará. Revista Brasileira de Ciência Veterinária, Niterói, v. 9, p. 107-109, 2002.

MACHADO, G. V.; SANTOS, B. S. Topografia do cone medular no Tamanduá-mirim (Tamandua tetradactyla Linnaeus, 1758) (Xenarthra: Myrmecophagidae). Archives of Veterinary Science, Curitiba, v. 13, n. 3, p. 172-175, 2008.

MOSKOSKI, J. H. Cães selvagens brasileiros-Cachorro-do-matode-orelhas-curtas. 2010. Disponível em: <http://vidaselvagem. spaceblog.com.br/310121/Caes-Selvagens-Brasileiros-Cachorrodo-mato-de-orelhas-curtas/>. Acesso em: 28 ago. 2010. 
SANTIAGO, W. Esqueletopia do cone medular em Canis familiaris. Arquivo da Universidade Federal Rural Rio de Janeiro, Seropédica, v. 4, p. 67-69, 1974.

SANTOS, A. L. Q.; LIMA, E. M. M. Topografia do cone medular em ovinos sem raça definida (Ovis aries Linneus, 1758). Ars Veterinária, Jaboticabal, v. 16, n. 3, p. 154-157, 2000.

SANTOS, A. L. Q.; LIMA, E. M. M.; SANTANA, M. I. S. Topografia do cone medular em caprinos da raça Saanen. Arquivos de Ciências Veterinárias e Zoologia da UNIPAR, Umuarana, v. 4, n. 1, p. 25-29, 2001.
SCAVONE, A. R. F.; GUIMARÃES, G. C.; RODRIGUES, V. H. V.; SASAHARA, T. H. C.; MACHADO, M. R. F. Topografia do cone medular da paca (Agouti paca, Linnaeus - 1766). Brazilian Journal of Veterinary Research and Animal Science, São Paulo, v. 44, suplemento, p. 53-57, 2007.

SILVA, P. H. C.; SILVA, R. M.; LIMA, E. M. M. Topografia do cone medular em gatos sem raça definida. Arquivo Brasileiro de Medicina Veterinária e Zootecnia, Belo Horizonte, v. 61, n. 5, p. 1062-1066, 2009. 